\title{
Surgical treatment of hiatal hernia, with particular reference to the transthoracic subdiaphragmatic approach
}

\author{
S. ZEL L OS \\ From the Regional Cardiothoracic Unit, Newcastle upon Tyne
}

In the past 15 years rapid advances have been made in the diagnosis and surgical treatment of diaphragmatic hernia, particularly the acquired oesophageal type. The masterly elucidation of the entire subject by Barrett (1950), Allison (1951), Sweet (1952), and others has been responsible for raising this subject from the realm of a surgical curiosity to the forefront as a subject of great surgical interest, occasionally beset with problems of the utmost difficulty.

Allison and Sweet prefer the transthoracic approach because it is easier to repair the hiatus from the thorax. Though the superiority of the transthoracic over the abdominal approach to the hiatus is generally recognized, the presence of associated lesions in the upper abdominal cavity is, in the opinion of many, a valid reason for the abdominal approach.

We are of the opinion that the most satisfactory approach to the hiatus is the transthoracic subdiaphragmatic approach, as introduced by Logan, which fulfils the two essential criteria of free access to the upper abdomen and easy access to the diaphragmatic hiatus and thoracic cavity.

In the Newcastle, England, Regional Cardiothoracic Unit, during the period 1951-64, a series of 800 patients with symptoms of reflux oesophagitis were investigated and treated. The purpose of this paper is to describe a new approach to the hiatus and to discuss the clinical features, diagnosis, surgical treatment, and results in this series.

\section{INCIDENCE}

Oesophageal hiatal hernia is the most common type of diaphragmatic hernia. Allison (1961) found that 10 to $12 \%$ of patients who underwent gastrointestinal tests had a hiatal hernia. Undoubtedly, many people have symptoms of hiatal hernia or 1Present address : Georgetown University Hospital, Washington,
D.C., U.S.A. suffer from mild dyspepsia and never seek treatment. The increasing frequency with which this condition is recognized at present may be attributed to the constant awareness of the members of the medical profession.

Herniae through the oesophageal hiatus are of two main varieties: the sliding type (Fig. 1), also referred to as the short oesophagus, and the paraoesophageal or rolling variety (Fig. 2). A third type, which is a combination of the two previously mentioned, is also recognized. The hernia is usually acquired, although some are observed in infancy.

The sliding hernia is by far the predominant type. It was present in $90 \%$ of the 800 patients. The paraoesophageal type was observed in almost $9 \%$.

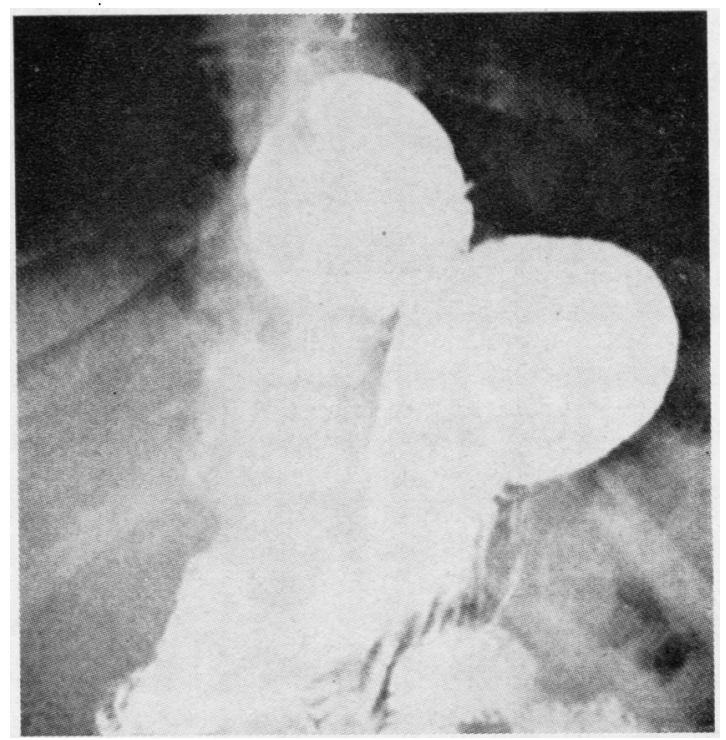

FIG. 1. Barium swallow showing a hiatal hernia of the sliding variety. Note the oesophagogastric junction well above the diaphragm. 

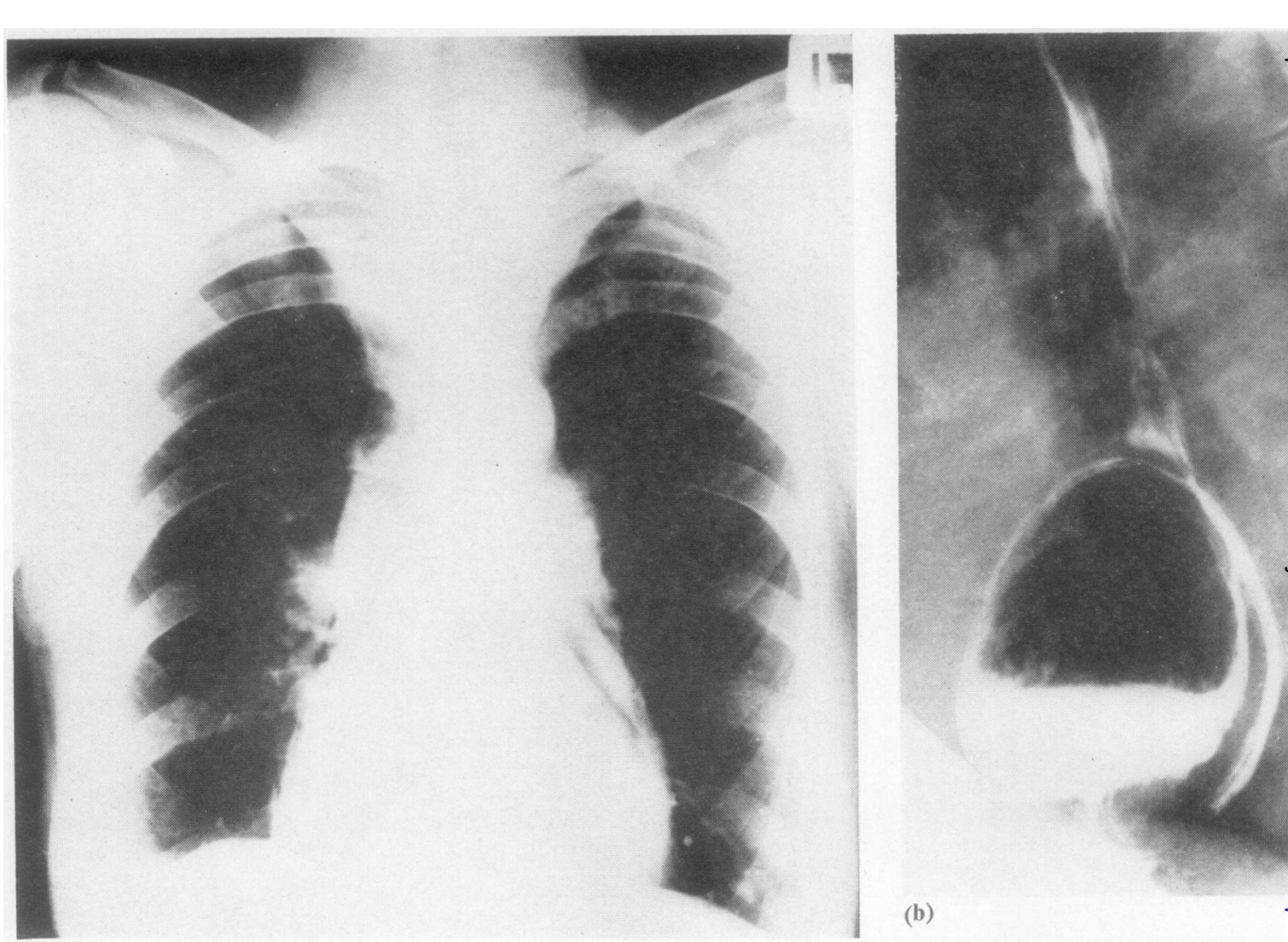

(b)

(a)

FIG. 2 (a) Postero-anterior view of the chest showing paraoesophageal hiatal hernia. (b) Barium swallow of the same $\stackrel{0}{x}$ patient showing typical example of paraoesophageal hernia with competent cardia. Note the gastric pouch in front of the $\frac{0}{3}$ oesophagus.

Sliding hiatal hernia as a result of trauma has been reported by Harrington (1952) and Marchand (1962), and it was present in one of our patients in the present series following an automobile accident. A repair of the hiatal hernia three months later gave satisfactory results.

The percentage of paraoesophageal herniae in our series is lower than in the series reported by Allison (1951) and Collis (1961), and higher than in the series reported by Sweet (1962).

\section{SEX AND AGE INCIDENCE}

The sex incidence followed the pattern reported by Humphreys, Ferrer, and Wiedel (1957) and Nygaard, Linaker, and Helsingen (1964). There were 576 females and 224 males, or a ratio of $2: 1$.

Ages varied from 2 to 74 years. Fifty-four herniae occurred in children under 10 years.

\section{CLINICAL FEATURES}

The symptoms were usually related to the particular $D$ type of hernia. In the sliding type, the symptoms were due to reflux oesophagitis caused by the re- $N$ gurgitation of gastric contents into the oesophagus through an incompetent cardia.

Pain, heartburn, and dysphagia, occurring either constantly or intermittently, were the most com mon symptoms in this series. Many complained ofo high epigastric pain aggravated by stooping or $\bar{\varnothing}$ lying flat. In some, the pain radiated to the back $\stackrel{?}{+}$ between the shoulder blades, to the neck or arms, $\underset{T}{0}$ often awakening the patient in the middle of the $\frac{0}{\mathbb{D}}$ night, after which the patient obtained relief by sitting up or sleeping in a propped-up position. This $\stackrel{\mathbb{Q}}{\mathbb{Q}}$ nocturnal aggravation of symptoms was due to aত̃ reduction in saliva during the night and the horizontal position of the patient. Heartburn witho 
regurgitation of acid material, which the patient described as vomiting, was a common symptom.

The majority complained of intermittent dysphagia. This may occur before stenosis has developed and is most probably due to spasm of the lower oesophagus as a result of reflux of gastric contents. Later, dysphagia becomes constant and progressively worse as stenosis develops. Twentythree patients had symptoms of chronic pneumonitis as the result of aspiration. Forty-seven patients had a history of either frank melaena or haematemesis. Another 34 had severe secondary anaemia. The outstanding clinical feature in all the children was effortless vomiting; malnutrition with haematemesis or melaena was present in $10 \%$ of these children.

In the paraoesophageal type, the patients usually had vague dyspeptic symptoms, including fullness after meals and flatulence. Some complained of palpitation and shortness of breath. Six patients were admitted for investigation and treatment of melaena. Two were treated on an emergency basis because of volvulus of the intrathoracic stomach.

Either occult or overt bleeding with resulting secondary anaemia occurred in both types, but predominantly in the sliding variety in a ratio of $42: 6$. The incidence of anaemia alone in only 81 of our patients is lower than that reported by Murphy and Hay (1943), DeVito, Listerud, Nyhus, Merendino, and Harkins (1959), and Johns and Clements (1961). The incidence in their respective series varied from $18 \%$ to $30 \%$.

The size of the hiatal hernia had no definite relation to the severity of symptoms, and all 11 patients with an incompetent cardia and no radiologically proven hernia had severe symptoms.

\section{DIAGNOSIS}

The clinical history is usually suggestive of the diagnosis of hiatal hernia, but occasionally difficulties arise in the differential diagnosis from angina attacks, cholelithiasis, peptic ulcer, and chronic pancreatitis. A barium swallow performed during an attack in a patient with doubtful electrocardiographic changes of infarction may be of considerable diagnostic value.

Radiological and oesophagoscopic examinations are the two important means of confirming the diagnosis of hiatal hernia.

\section{RADIOLOGICAL EXAMINATION}

It is important to make a thorough examination of the oesophagus, stomach, and duodenum to exclude other lesions, e.g., oesophageal or gastric neoplasm and duodenal ulcer with gastric retention.

The patient must be examined in the erect, recumbent, head-tilted-down position for anteroposterior and lateral screening and radiography. Unless the herniation and reflux are immediately seen, steps must be taken to increase the intraabdominal pressure by palpation, elevation of the extended legs, and coughing. Drinking in the Trendelenburg position may also be tried. In spite of all efforts, a hernia or reflux may not be seen at one examination but could be demonstrated at a subsequent one.

Although routine radiological examination usually demonstrates a hiatal hernia, experience has shown that in a small number of patients this cannot be achieved in spite of the fact that the clinical symptoms are strongly suggestive of reflux oesophagitis. In the present series we had 11 patients in whom we failed to demonstrate the hiatal hernia radiologically.

The studies of Ellis, Code, and Olsen (1960) and the studies of our own clinical staff showed that a manometric technique of measuring pressures and $p \mathbf{H}$ at the oesophagogastric junction was useful in the detection of small hiatal herniae that cannot be demonstrated by routine radiological examination.

\section{OESOPHAGOSCOPY}

We consider oesophagoscopy and oesophageal biopsy as indispensable diagnostic procedures both in confirming the diagnosis and evaluating the degree of oesophagitis. For this reason, oesophagoscopy was undertaken in almost every patient with hiatal hernia and revealed oesophagitis in 450 patients with sliding hiatal hernia. In 82 the pathological process had resulted in stricture formation (Fig. 3). Two patients had peptic ulcers, and biopsy showed columnar epithelium. In the paraoesophageal variety only the six patients with the slide had oesophagitis. We found a marked discrepancy in the world literature as to the frequency of peptic oesophagitis in hiatal hernia, the figures ranging from $3 \%$ to $87 \%$. This may be explained by differing criteria in the diagnosis of oesophagitis.

We noted that in children the pathological process of reflux oesophagitis was more rapid, and within a short period from the onset of symptoms a stricture may have formed, destroying part of the oesophageal wall. Fourteen out of 54 children developed such a stricture. The pathological findings due to reflux oesophagitis found during oesophagoscopy in the present series are as follows: minimal oesophagitis, 211 ; moderate oesophagitis, 


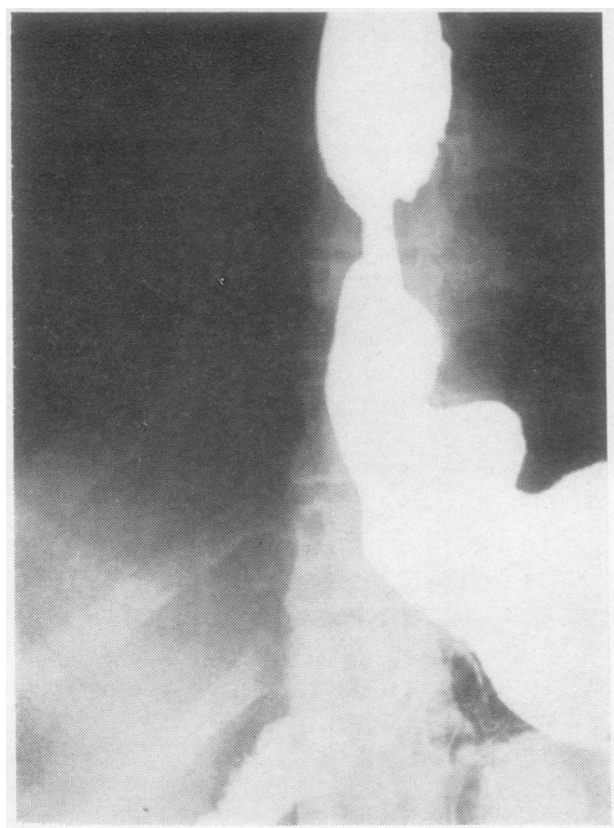

(a)

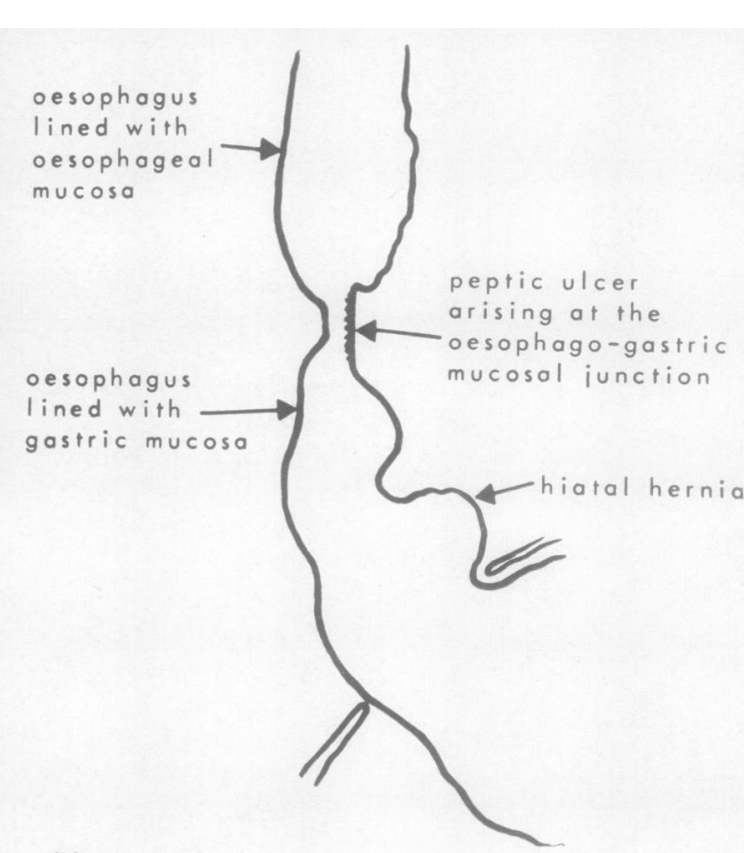

(b)

FIG. 3 (a) Barium swallow showing peptic oesophageal ulcer and stricture formation. oesophagus as seen at oesophagoscopy and at operation.

89 ; gross oesophagitis, 68 ; oesophagitis with stenosis, 82 ; and no oesophagitis, 350 .

\section{ASSOCIATED LESIONS}

Patients with a hernia through the oesophageal hiatus may have other associated lesions, including gastric or duodenal ulcer, chronic cholelithiasis, and pancreatitis. Associated lesions were present in $3 \%$ or 24 patients. However, Sweet (1962) reported an incidence of $25 \%$ and DeBakey (1963) $30 \%$ in their respective series.

A thorough examination of the patient is necessary before surgery to prevent mistakes in diagnosis and to determine whether there is another lesion in the stomach or duodenum causing delay in gastric emptying, which in turn produces reflux oesophagitis. The following examples are illustrative.

In one patient three lesions were coexistent: hiatal hernia, cholelithiasis, and coronary insufficiency. A repair of the hernia, cholecystectomy, and Beck's operation at the same time gave satisfactory results. In two patients hiatal hernia was associated with cholelithiasis. Both lesions were operated upon through the same approach with excellent results. In two patients a peptic ulcer associated with the hiatal hernia was severe enough to require partial gastrectomy at the same time. In 13 patients vago-0. tomy and pyloroplasty or gastroenterostomy were done simultaneously. In two, achalasia of the oeso-o phagus and hiatal hernia coexisted. This is a rare combination, but repair of the hernia and ao Heller's operation gave satisfactory results.

The incidence of cardiospasm in combinationo with hiatal hernia is reported differently by various 3 authors. Allison (1951) found an incidence of 3\%.윽 It is conceivable that the cardia becomes incom petent as a result of repeated dilatations or excessive traction of the oesophagogastric junction during Heller's operation.

Four patients presented with a diverticulum of the lower oesophagus as well as hiatal hernia. Ex $\frac{N}{\mathrm{~N}}$ cision of the diverticulum and repair of the herniag were carried out simultaneously.

\section{TREATMENT}

Medical treatment of hiatal hernia with refluxo oesophagitis is indicated only when the symptoms are mild and the oesophagitis minimal. It is impor $-\vec{D}$ tant to perform a repeat oesophagoscopy, even if there is clinical improvement under this regimen. In the experience of this unit, peptic oesophagitis is 
usually a progressive condition. There is always a danger of irreversible damage to the oesophageal mucosa while medical treatment is used.

In the paraoesophageal type of hiatal hernia, although the symptoms tend to be relatively mild, there are dangers. A thoracic stomach can develop peptic ulceration, with the attendant complications of severe haematemesis or perforation. Volvulus obstruction of the intrathoracic stomach can occur at any time. Two of our patients were admitted as emergencies for volvulus of an intrathoracic stomach; one died post-operatively. Paraoesophageal hernia associated with melaena was the indication for surgery in six patients of the present series.

\section{INDICATIONS FOR SURGERY}

Because of the low operative mortality rate, the availability of a satisfactory operative procedure and the remarkably good ${ }^{1}$ long-term results, we advise surgery in all patients with reflux oesophagitis or paraoesophageal hernia.

The chief indications for surgical intervention in the present series were failure of medical treatment and the presence of oesophagitis and/or its complications (haemorrhage, haematemesis, or melaena, anaemia, or stricture formation). Thus, our policy regarding the surgical treatment of hiatal hernia has been as follows : for sliding hernia with reflux oesophagitis and no stricture-reduction of the hernia and repair of the hiatus; for hernia with reflux oesophagitis and a dilatable stricture-reduction of the hernia, vagotomy, pyloroplasty, and dilatation of the stricture at intervals, if necessary ; for sliding hernia associated with tough fibrous stricture and considerable shortening of the oesophagus-resection of the stricture and replacement by stomach, jejunum, or colon; for incompetent cardia with oesophagitis without radiological demonstration of a hiatal hernia-repair, as for sliding hiatal hernia ; for paraoesophageal hernia-reduction of the hernia and repair of the hiatus.

\section{SURGICAL TECHNIQUES}

Of the 800 patients investigated, 600 underwent surgery. The other 200 patients were considered unfit for operation, had symptoms too mild to warrant operation, or refused surgery.

Five hundred and thirty-nine patients underwent operations for hiatal hernia of the sliding variety and 61 for hiatal hernia of the paraoesophageal type through the transthoracic approach.

'Many informed surgeons disagree with this statement.-EDrror.
All patients were operated upon by members of the full-time staff and myself, with fairly constant operative techniques.

Three different techniques were used; accordingly, the patients can be divided into three groups.

TECHNIQUE NO. I This technique, used between 1951 and 1957 on 101 patients, was devised by Mason and has been described in detail by Evans and Simpson (1950). The approach is by a left thoracotomy incision through the bed of the eighth rib. The hernial sac is identified. An incision planned to preserve the inner branches of the phrenic nerve is made in the diaphragm ; the hiatus is approached from the abdominal aspect ; and reduction and repair are effected from below the diaphragm. The hernial contents are freed from the sac and a rubber catheter is passed as a sling around the oesophagogastric junction. The enlarged oesophageal hiatus is repaired with interrupted no. 4-0 linen sutures. The oesophagus is anchored to the reconstructed hiatus with a few stitches.

These patients have been followed for from four to 10 years, and 11 had recurrence of the hernia, two had gross reflux, three had moderate reflux with minimal symptoms, and six had slight reflux without symptoms. In the remainder, the results were good.

TECHNIQUE NO. II During the period 1955-64, a second technique described by Allison (1951) was used on 429 patients. This group of patients was followed for from six months to six years, and in 27 the hernia has recurred, in four there was gross reflux with symptoms, in eight there was moderate reflux with minimal symptoms, and in 12 there was slight reflux without clinical symptoms.

TECHNIQUE NO. III This approach was introduced by Logan in Edinburgh and has been followed by Mason since 1958. Hitherto it has not been published. The technique of hiatal repair is similar to that described by Nissen (1956).

The left chest cavity is entered through the bed of the ninth rib. The incision passes well forward, dividing the costal margin through the ninth costal cartilage, if necessary. The rib is not resected. This gives an excellent exposure of the left hemidiaphragm. The incision of the diaphragm is considered to be of special importance, and this is a modification of the previous techniques. The diaphragm and its pleural covering are incised parallel to and about $1.5 \mathrm{~cm}$. from the costal margin, beginning in the rib bed anteriorly. The diaphragmatic incision extends forward and medially to within 4 to $5 \mathrm{~cm}$. of the sternum and backward and laterally 

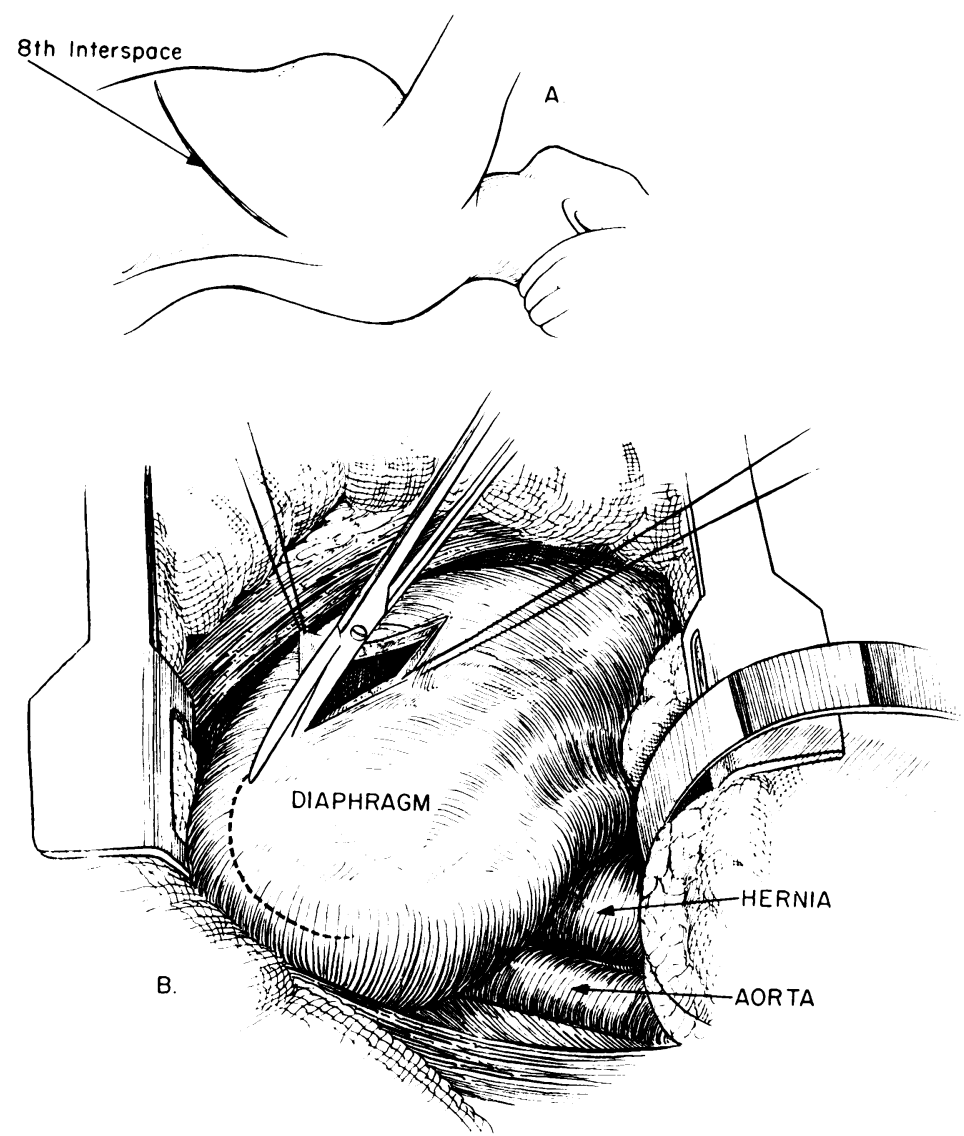

FIG. 4. (A) The line of the thoracotomy incision through the bed of the ninth rib. (B) The line of the dia-윽 phragmatic incision according to Logan's technique.

until the peritoneum is replaced by perinephric fat. This initial incision does not include peritoneum, which is now divided over the same length, but about $3 \mathrm{~cm}$. from the costal margin (Fig 4). The larger peritoneal cuff on the costal side of the diaphragmatic incision facilitates closure. This incision of the diaphragm does not damage the phrenic nerve, and post-operatively the diaphragmatic movements are normal.

The hiatal oesophagus can now be approached from below the diaphragm as well as from above it. The stomach and duodenum are examined for the presence of an ulcer or any other abnormality. The gall bladder is palpated also for stones and, if necessary, cholecystectomy can be performed. The dissection of the hiatus is carried out from the abdominal side (Fig. 5). The left margin of the right crus is dissected first. The peritoneal reflection together with the phreno-oesophageal ligament are divided and the oesophagus can be dissected and exposed distinctly all round. A piece of rubber tubing is passed around the oesophagus for traction.
The dissection of the right crus and the entire hiatus is now completed. Vagotomy is performed at this stage, if necessary; otherwise, the vagus nerves are carefully preserved.

By traction on the rubber tubing, the cardia is $\frac{\text { ? }}{2}$ drawn into its normal position below the dia- $\rightarrow$ phragm. The anaesthetist is asked to pass a moderate-sized stomach tube. With this in position, the $N$ margins of the enlarged hiatus are approximated behind the oesophagus with interrupted linen 0 sutures (Fig. 6). In our experience, repair of the hiatus may be made more secure if it is made tight. The patient will usually have mild post-operative 0 dysphagia ; however, this generally subsides within a week. In the long run, we have noted this prevents a recurrence of oesophageal reflux. The cardia is 0 next invaginated into the stomach, inkwell fashion, as described by Nissen (1956) (Fig. 6, D, E).

The central portion of the diaphragm is re- $\mathbb{D}$ attached to the peripheral cuff left of the costal 0 margin, using interrupted silk sutures at $1 \mathrm{~cm}$. intervals. Pleura, muscle, and peritoneum are included 


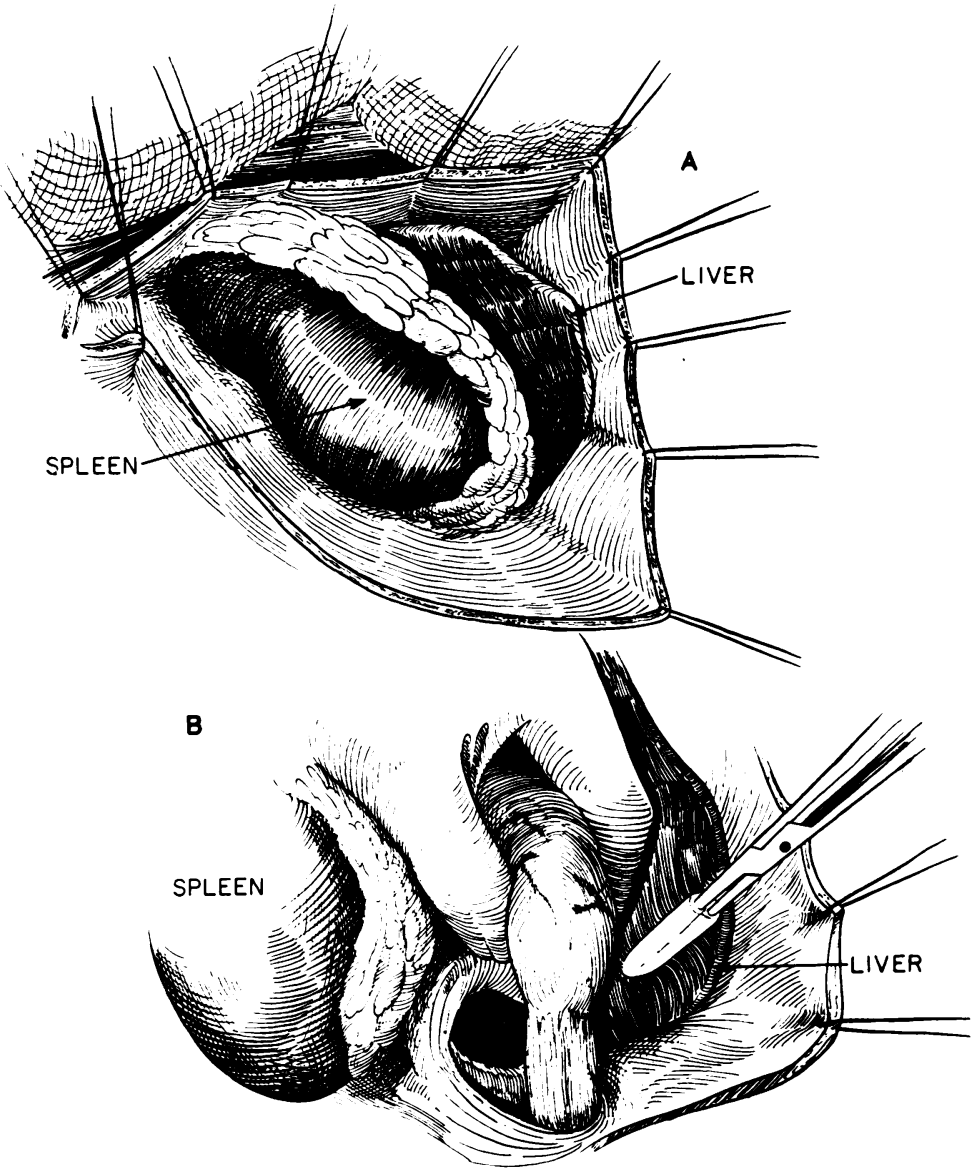

FIG. 5. (A) Shows the excellent exposure of the upper abdomen. (B) Shows the ease with which the hiatus is dissected tirrough this approach.

in each suture. The pleural cavity is drained and the chest closed in layers.

We used this approach in 70 subsequent patients and five patients had a recurrence, one had gross reflux, three developed moderate reflux without anatomical recurrence of the hernia, and four had minimal reflux without symptoms. In the remainder the results were excellent.

\section{FOLLOW-UP}

It has been our practice to follow every patient operated on at regular intervals for years, if necessary. The follow-up period in the present series ranged from six months to 14 years. All our patients have a post-operative barium meal before discharge from hospital, and repeated oesophagoscopical and radiological examinations are carried out if symptoms recur.

In the follow-up studies the patients fell into two categories-those with minimal or moderate gastric reflux seen at radiological examination two weeks after surgery and those with anatomical recurrence of the hernia or gross oesophageal reflux seen from six months to three years or longer post-operatively.

It is evident that the surgical treatment of the first group of patients achieved only the anatomical objective but failed to restore the normal physiology of the cardia. In the second, surgery achieved neither. Subsequently, all patients belonging to the second category submitted by necessity to a second operation, whereas the patients in the first category were treated conservatively with the required medical treatment only.

\section{MORTALITY AND MORBIDITY}

In the whole series there have been 14 deaths, the overall post-operative mortality rate being $3 \%$. Five patients died of pulmonary embolism, two of coronary thrombosis, two of necrotic enteritisone on the third post-operative day and the other 


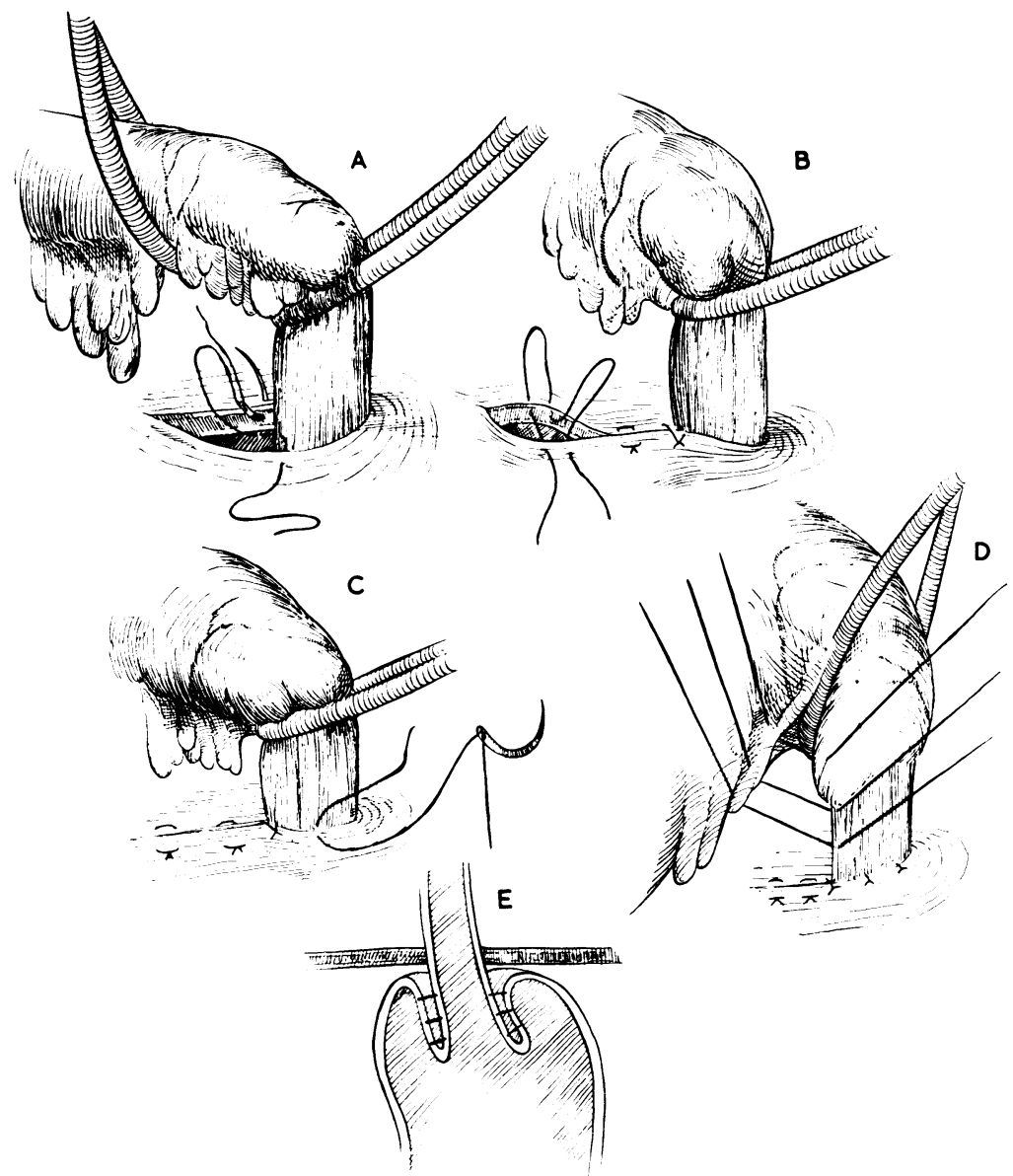

FIG. 6. (A) (B) The technique of 은 hiatal repair. (C) Fixation of the oesophagus to the diaphragmatic $\subseteq$ hiatus. (D) (E) Inkwell invagination of the cardia according to Nissen's technique.

three weeks after surgery. A 60-year-old patient died of renal failure. A patient died of empyema two weeks after surgery. Two patients, aged 62 and 71 respectively, died of bilateral bronchial pneumonia. A 55-year-old patient with peptic stricture of the oesophagus and duodenal ulcer developed peritonitis and shock 24 hours after surgery. She had undergone a hiatal hernia repair, vagotomy, and pyloroplasty. In spite of early recognition of the complication, her condition deteriorated so rapidly that it did not allow exploration; she died the same day. Necropsy showed that the causes of death were perforation of a duodenal ulcer and bilateral, unexplained suprarenal haemorrhage.

\section{DISCUSSION}

Reflux oesophagitis is a well-recognized clinical entity which has received increasing attention in recent years. Accumulated experimental and clinical data suggest that it is caused by malfunction $\delta$ of the oesophagogastric junction resulting in gastric reflux of acid-pepsin into the oesophagus.

Although the mechanism of gastric reflux $\supset$ through the cardia in a normal individual is still not well understood, certain factors have been $\bar{N}$ shown to play an important part in the mainten-o ance of a competent cardia: the sphincter-like $N$ action of the circular muscle fibres of the lower end $\mathbb{\omega}_{\omega}^{N}$ of the oesophagus (this lower portion of the oeso- -0 phagus is under vagal control, inhibition being mediated by impulses transmitted intramurally); the oblique entry of the oesophagus into the stomach ; the sling action of the right crus, which 0 during contraction helps to increase the angle be- ${ }^{\circ}$ tween the oesophagus and the stomach; and $a \stackrel{\mathbb{}}{\mathbb{P}}$ valve at the cardia, described by Dornhorst, Harri-ब्ष son, and Pierce (1954), which is supposed to consist of a series of mucosal folds surrounding the cardia, and resembling a rosette. 
In the sliding hiatal hernia all the above factors are disorganized, particularly the oblique entry of the oesophagus into the stomach and the sling action of the right crus. In the paraoesophageal type the important factor, namely the oblique entry of the oesophagus into the stomach, is unaffected and reflux does not take place. Thus the objective of surgical treatment is to restore to normal those factors responsible for producing the hiatal hernia.

To this end, various techniques have been described by different workers. Harrington (1948) suggested an abdominal approach, reduction of the hernia, and repair of the hiatus, which is reinforced with fascia lata. For irreducible herniae he has advocated phrenic crush. Allison (1951) has introduced a transthoracic approach, division of the phreno-oesophageal ligament, which is then stitched to the undersurface of the diaphragm, and approximation of the two limits of the right crus behind the oesophagus. He reported a recurrence rate of $3 \%$. However, Stensrud (1954) and Husfeldt, Andreassen, Lindenberg, and Thomsen (1960) demonstrated radiological recurrence in $38 \%$ of their patients after an Allison type of repair. This discrepancy in figures may result from the use of different criteria on the radiological examinations.

Sweet (1962), using a transthoracic approach, crushed the phrenic nerve, dissected and excised the hernial sac or plicated it, and repaired the hiatus by means of sutures of heavy silk placed in the diaphragmatic muscle on either side of the oesophagus. His figures show that of a total of 394 patients, 31 had proven recurrences and three had only partial relief of the symptoms. Nissen (1956) described an abdominal approach, repair of the hiatus behind the oesophagus, inkwell invagination of the cardia into the oesophagus, and gastropexy.

In our institution, surgical treatment of hiatal hernia has passed through three phases during the years 1951 to 1964. Between 1951 and 1957 we followed the technique used by Mason. Follow-up has shown that after this type of operation the recurrence rate was $10 \%$. In view of this, between 1954 and 1964 we used the technique described by Allison (1951), and it was found that the recurrence rate was virtually the same. For this reason the Logan-Nissen technique has been employed since 1958 , and we were much impressed by the excellent approach to the hiatus, lower chest, and upper abdomen. Using this technique we have been able to reduce the recurrence rate to just below $8 \%$.

We consider Logan's approach to the hiatus to be superior to the other approaches for the following reasons: repair of the hiatal hernia can be achieved with more precision and less difficulty than with the abdominal approach ; intrathoracic lesions can be dealt with at the same time as repair of the hiatal hernia ; it facilitates the management of concurrent intra-abdominal lesions in a single operation; this approach is ideal for a recurrence of hiatal hernia when technical faults, adhesions from the previous operations, and distortion of the anatomy of the hiatus demand an excellent approach to allow for adequate exploration ; resection of the lower oesophageal stricture or hernia, with a high intrathoracic cardia requiring mobilization of the oesophagus, can be accomplished without a thoraco-abdominal incision; coexisting coronary insufficiency can be dealt with at the same time; it was found on fluoroscopy that the postoperative function of the diaphragm after this type of repair remains normal ; the convalescent period is no longer than when the abdominal approach is used, or approximately one week.

A careful analysis of the operative findings in 41 patients with a recurrence of the hernia has shown, as could be expected, that there were three main factors responsible for this recurrence: elevation of the cardia above the diaphragm, patulous hiatus, and abolition of the oesophagogastric angle.

Experience has shown that in a number of patients with sliding hiatal hernia difficulty is encountered in bringing the cardia below the diaphragm, the hernia being repaired under tension. Thus, repair of the sliding variety of hernia is more difficult technically than that of the paraoesophageal type because of the need to prevent reflux of acid gastric contents into the oesophagus and to place the gastro-oesophageal junction below the diaphragm. These two requirements are probably related. In these difficult situations, transthoracic mobilization of the oesophagus as high as necessary may be imperative in order to achieve one of the two essential principles in the hiatal repair, the subdiaphragmatic placement of the oesophago. gastric junction.

Obesity and poor development of the right crus may be contributing factors toward recurrence. The value of the phreno-oesophageal ligament as fixing agent is disputed. Allison (1951), Hayward (1961), and Paulson, Shaw, and Kee (1962), among other surgeons, consider the meticulous and careful suture of the phreno-oesophageal ligament to the diaphragm to be a very important step in the repair of the hiatus, whereas Harrington (1952) and Sweet (1962) disregard it. Barrett (1954), Hill, Chapman, and Morgan (1961), and Lortat-Jacob, Dromer, Lebas, Maillard, Richard, and Fekete (1962), among others, placed considerable emphasis on the reconstruction of the angle of His.

Nissen (1956) advocated the inkwell type of invagination of the cardia. It is of interest to note 
that in one patient who had a Logan-Nissen type of repair and recurrence of the hernia we found at operation a patulous hiatus, but the inkwell invagination of the cardia was intact. Though it is difficult to draw conclusions from only one recurrence, the presence of gastric reflux in five patients at the follow-up studies makes us sceptical as to whether this type of invagination of the cardia alone controls the gastric reflux.

On the basis of the material described in this study, it is our opinion that the most important steps in hiatal hernia repair are: placement of the oesophagogastric junction well below the diaphragm and reduction of the hernia without tension, careful approximation of the two limbs of the right crus by means of interrupted sutures, and reconstruction of the angle of His with interrupted stitches.

It has been found that, even after proper anatomical repair of the hiatus, post-operative reflux occurs occasionally. In this small group of patients pre-operative gastric acidity studies are necessary and, in the presence of hyperacidity, we feel that the operation should be combined with vagotomy and pyloroplasty. Few patients in the present series have been treated successfully in this way.

\section{SUMMARY}

A review of the pattern of illness, management, and complications of 800 patients with reflux oesophagitis indicates that medical treatment should be employed only in those who are unfit for surgical repair or who refuse surgery. When peptic stricture is present, one may try dilatation first, and, if no relief follows, resection and interposition of a loop of the jejunum or colon is the treatment of choice. The best post-operative results seem to be associated with a very tight oesophagogastric junction with radiological appearances comparable with cardiospasm. A transthoracic subdiaphragmatic operation has proved to be an excellent approach to the hiatus, upper abdomen, and lower chest. In our experience, this operation is the one of choice because of the ease with which the surgeon can deal with frequently encountered intrathoracic and intra-abdominal lesions in a single operation.

\section{REFERENCES}

Allison, P. R. (1951). Reflux esophagitis, sliding hiatal hernia, and the anatomy of repair. Surg. Gynec. Obstet., 92, 419.

(1961). Swallowing and dysphagia. J. roy. Coll. Surg. Edin., 6, $\vec{\circ}$ 113.

Barrett, N. R. (1950). Chronic peptic ulcer of the oesophagus and 'oesophagitis'. Brit. J. Surg., 38, 175.

-(1954). Hiatus hernia: A review of some controversial points. Ibid., 42, 231.

Collis, J. L. (1961). A review of surgical results in hiatus hernia. Thorax, 16, 114.

DeBakey, M. E. (1963). In The Year Book of General Surgery, 196361, p. 425. Year Book Medical Publishers, Chicago

DeVito, R. V., Listerud, M. B., Nyhus, L. M., Merendino, K. A. and Harkins, H. N. (1959). Hemorrhage as a complication of reflux esophagitis. Amer. J. Surg., 98, 657.

Dornhorst, A. C., Harrison K., and Pierce, J. W. (1945). Observations on the normal oesophagus and cardia. Lancet, 1, 695.

Ellis, F. H., Jr., Code, C. F., and Olsen, A. M. (1960). Long esophagomyotomy for diffuse spasm of the esophagus and hypertensive gastroesophageal sphincter. Surgery, 48, 155.

Evans, C. J., and Simpson, J. A. (1950). Fifty-seven cases of dia phragmatic hernia and eventration. Thorax, 5, 343.

Harrington, S. W. (1948). Various types of diaphragmatic hernia treated surgically. Report of 430 cases. Surg. Gynec. Obstet., 86, 735 .

(1952). Esophageal hiatus diaphragmatic hernia. Rocky Mtn med. $J ., 49,665$.

Hayward, J. (1961). The phreno-oesophageal ligament in hiatal hernia repair. Thorax, 16, 41.

Hill, L. D., Chapman, K. W., and Morgan, E. H. (1961). Objective evaluation of surgery for hiatus hernia and esophagitis. J. thorac. cardiovasc. Surg., 41, 60.

Humphreys, G. H., Ferrer, J. M., Jr., and Wiedel, P. D. (1957) Esophageal hiatus hernia of the diaphragm. An analysis of surgical results. J. thorac. Surg., 34, 749.

Husfeldt, E., Andreassen, M., Lindenberg, J. L., and Thomsen, G. (1960). Operative treatment of sliding hiatal hernia in adults. Acta chir. scand., Suppl. 253, 33.

Johns, T. N. P., and Clements, E. L. (1961). The relief of anemia by repair of hiatus hernia. J. thorac. cardiovasc. Surg., 41, 737.

Lortat-Jacob, J. L., Dromer, M., Lebas, P., Maillard, J. N., Richard, C., and Fekete, F. (1962). A propos de 221 interventions pour hernie par l'hiatus chez oesophagien l'adulte. Ann. Chir., 16, 985.

Marchand, P. (1962). Traumatic hiatus hernia. Brit. med. J., 1, 754.

Murphy, W. P., and Hay, W. E. (1943). Symptoms and incidence of anemia in hernia at the esophageal hiatus. Arch. intern. Med., 72, 58 .

Nissen, R. (1956). Die Gastropexie als alleiniger Eingriff bei Hiatushernien. Dtsch. med. Wschr., 81, 185.

Nygaard, K., Linaker, O., and Helsingen, N., Jr. (1964). Esophageal hiatus hernia, a follow-up study. Acta chir. scand., 128, 293.

Paulson, D. L., Shaw, R. R., and Kee, J. L. (1962). Esophageal hiatal diaphragmatic hernia and its complications. Ann. Surg., 155, 957.

Stensrud, N. (1954). Hiatus hernias. Acta chir. scand., 107, 57.

Sweet, R. H. (1952). Esophageal hiatus hernia of the diaphragm: the anatomical characteristics, technic of repair, and results of treatment in 111 consecutive cases. Ann. Surg., 135, 1.

(1962). Experiences with 500 cases of hiatus hernia. A statistical survey. J. thorac. cardiovasc. Surg., 44, 145. 